\title{
A valid treatment option for isolated congenital microgastria
} Mohammad Zamakhshary ${ }^{\mathrm{a}}$, Omar Oda ${ }^{\mathrm{a}}$, Abdullah Alrabeeah ${ }^{\mathrm{a}}$, Saud Al Jadaan ${ }^{a}$, Mohammad Al-Namshan ${ }^{a}$, Stanley Crankson ${ }^{\mathrm{a}}$, Mohammed Hasosah ${ }^{\mathrm{b}}$, Othman Aljohani ${ }^{\mathrm{b}}$ and Ahmed Abdulwahab ${ }^{\mathrm{c}}$

\author{
Congenital microgastria (CM) is an extremely rare anomaly \\ of the caudal part of the foregut. Treatment of $\mathrm{CM}$ has not \\ yet been standardized. We present the case of a 34-month- \\ old girl with an isolated $\mathrm{CM}$ complicated by nasogastric \\ tube-related gastric perforation. During the definitive \\ reconstructive surgery, a scarred structure $(1.5 \times 1.5 \mathrm{~cm})$ \\ was found to follow a dilated esophagus. The scarred \\ microstomach was resected, and a Roux-en-Y \\ esophagojejunostomy was performed. The patient has \\ been followed for 6 months. She tolerates a regular \\ oral diet and has reached acceptable growth parameters. \\ We describe the first case of $\mathrm{CM}$ to be treated with \\ resection of the microstomach and with a Roux-en-Y \\ esophagojejunostomy. Ann Pediatr Surg 7:114-116 ๔ \\ 2011 Annals of Pediatric Surgery
}

Annals of Pediatric Surgery 2011, 7:114-116

Keywords: congenital microgastria, gastrectomy, Roux-en-Y esophagojejunostomy

aDivision of Pediatric Surgery, Department of Surgery, King Fahad National Guard Hospital, King Saud Bin Abdulaziz University for Health Sciences, King Abdulaziz Medical City, Riyadh, ${ }^{b}$ Department of Pediatric Gastroenterology and 'Department of Pediatric Surgery, National Guard Hospital, King Abdulaziz Medical City, Jeddah, Kingdom of Saudi Arabia

Correspondence to Mohammad Zamakhshary, MD, MED, FRCSC, Division of Pediatric Surgery, Department of Surgery, King Fahad National Guard Hospital, King Abdulaziz Medical City, PO Box 22490, Mail Code: 1515, Riyadh 11426 , Kingdom of Saudi Arabia

Tel: + 966543427669 ;

e-mail: zamakhshary@gmail.com

Received 19 February 2011 Accepted 22 March 2011

complicated by an intraperitoneal leak from the site of jejunostomy that required two laparotomies. Later, the patient developed an enterocutaneous fistula that required a fourth laparotomy for repair. During this 7-month period, the patient was on total parenteral nutrition. When the patient recovered, minimal oral feeding with supplemental nasogastric tube feeding was administered. Despite aggressive nutritional support for 26 months, the repeated upper barium study and upper gastrointestinal (GI) endoscopy revealed a lack of gastric growth with no columnar mucosa. She was referred to us at the age of 26 months with a weight of $7.8 \mathrm{~kg}$. Laboratory results revealed a normal blood cell count with a hemoglobin level of $136 \mathrm{~g} / \mathrm{l}$ and a hematocrit of 0.416, normal renal and liver profiles, and a mild hypoalbuminemia of $34 \mathrm{~g} / \mathrm{l}$. Abdominal ultrasound showed normal liver, spleen, and kidneys. The echocardiography was normal. An upper GI contrast study revealed a dilated esophagus, a microstomach (Fig. 1), a high-grade gastroesophageal and intraesophageal reflux (from the dilated distal part to proximal part of the esophagus), and markedly delayed passage of the contrast material to the duodenum. An upper GI endoscopy with multiple biopsies revealed signs of esophagitis, lack of a visible stomach, inability to pass the scope to the duodenum, and the absence of gastric mucosa. The patient was put on prokinetic agents and was allowed minimal oral intake. Total parenteral nutrition was started to build up the patient as a preparation for reconstructive surgery, during which a very small scarred structure $(1.5 \times 1.5 \mathrm{~cm})$ was found distally to a dilated abdominal part of the esophagus. The scarred 'microstomach' was excised (Fig. 2a and b), and a Roux-en-Y esophagojejunostomy was performed. The pathology report described a scarred microstomach $(1.5 \times 1.5 \mathrm{~cm})$ with a normal pyloric 
mucosa at the distal end. Postoperatively, oral feeding was started gradually. The patient was discharged home 3 weeks after the surgery, tolerating $120 \mathrm{ml}$ of milk formula every $2 \mathrm{~h}$. The patient has been followed for 6 months Currently, she is 34 months old, with a weight of $11 \mathrm{~kg}$ (15th percentile) and is consuming a normal toddler's oral diet without supplementation.

\section{Discussion}

CM is a very rare disorder [1-7]. It was first reported in the 1800 s as a small, tubular, or saccular, incompletely rotated stomach associated with a megaesophagus [10].

Fig. 1

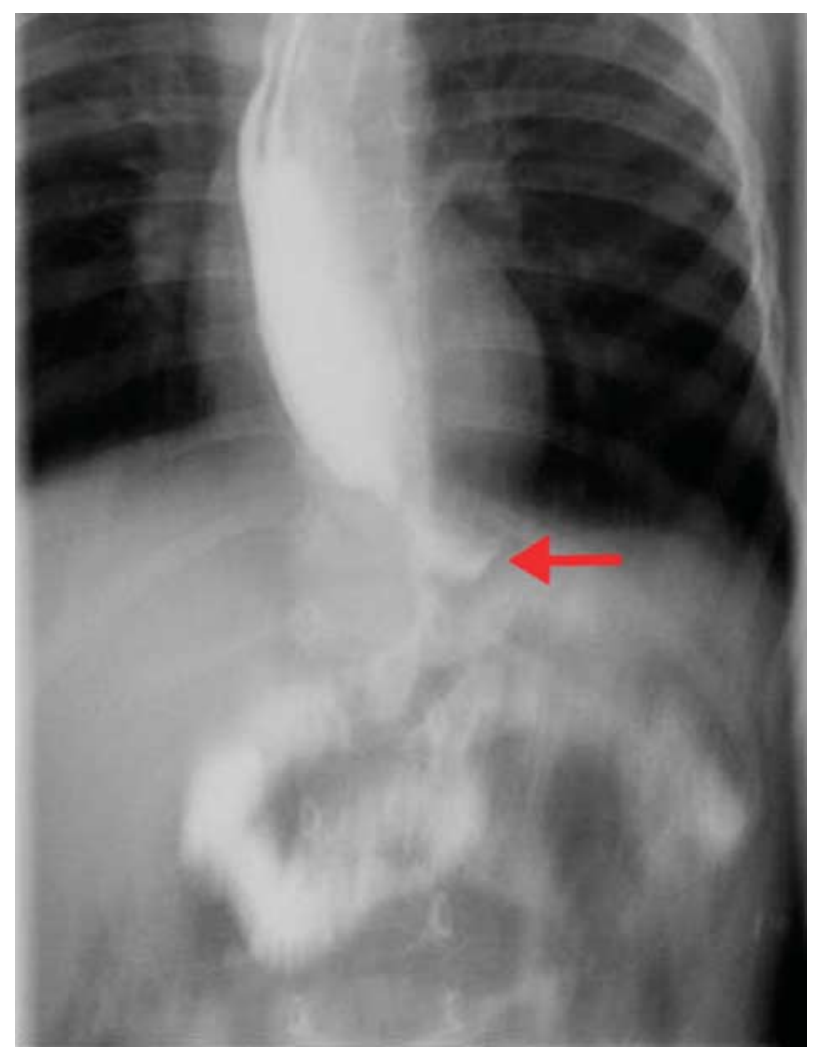

Upper gastrointestinal contrast study showing dilated esophagus with a microstomach (arrow).

Fig. 2

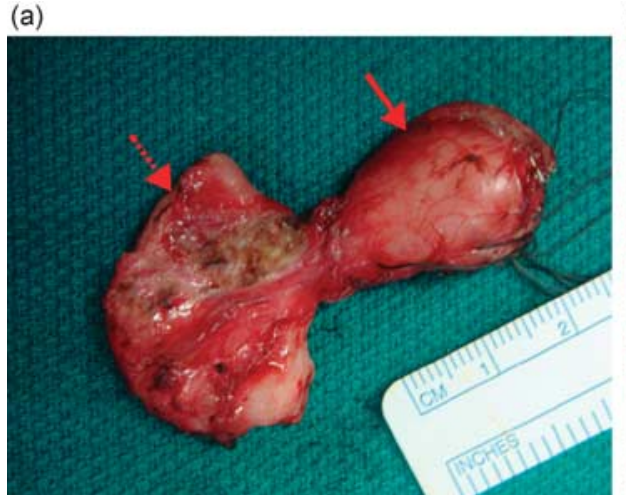

(b)

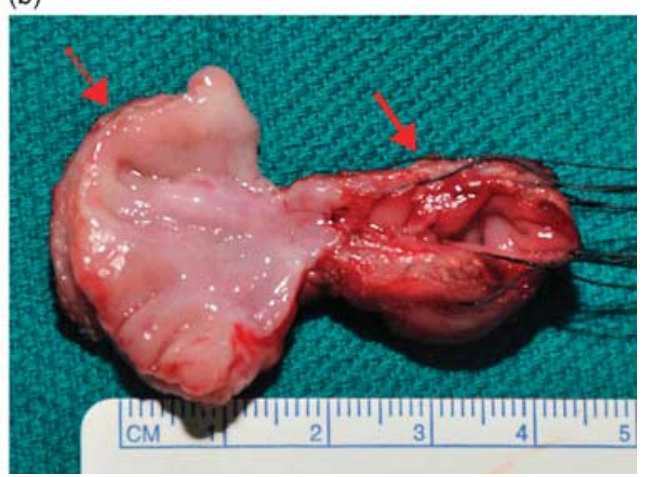

(a and b) Resected microstomach (arrow) with a part of the distal esophagus (dotted arrow).

A total of 59 cases were reported in the English literature before 2007 [3]. It is an early malformation of the distal foregut $[2,4,7]$. Associated malformations are very common, including asplenia; congenital heart disease; lung sequestration; laryngotracheal cleft; esophageal atresia; midgut malrotation; renal, limb, and central nervous system malformations; Pierre-Robin syndrome; and DiGeorge syndrome [2-5,7-11]. A genetic cause has been postulated [2,3,12]. Isolated $\mathrm{CM}$ is an extremely rare disorder, with only four cases reported [3]. Our patient had no associated anomalies. Prenatal diagnosis has been described; however, most cases presented in early infancy with nonbilious vomiting and failure to thrive $[1,3-7,12]$. Stridor is a rare presentation of CM [13]. The primary diagnostic modality is an upper GI contrast study. Treatment options include conservative management with frequent feeding of small amounts, which allow the stomach to enlarge over time, gastric augmentation with a jejunal loop (Hunt-Lawrence procedure), and total gastric dissociation with a Rouxen-Y esophagojejunostomy [3-7,12]. Jones and Cohen [3] stated that conservative treatment has shown poor results with poor somatic growth, sexual underdevelopment, and a delay in cognitive milestones, and recommended early reconstructive surgery. Jones and Cohen [3] reviewed 10 cases treated with the Hunt-Lawrence procedure and with a follow-up period ranging from 3 months to 18 years and reported good results. Total gastric dissociation was initially described by Lall in 2006 [9] as an alternative procedure for the management of gastroesophageal reflux in selected patients. Lall et al. [9] reported the use of total gastric dissociation for the treatment of three patients with insufficient stomach capacity, one of whom had severe CM in association with pure esophageal atresia; he reported excellent results of the procedure, their patients were able to eat normally without any food alteration or restrictions and to enjoy normal growth. However, the fate of the microstomach was not discussed in this study.

In our case, the failure of conservative treatment and the inability to perform gastric augmentation because of the very small scarred stomach directed us toward Roux-en-Y esophagojejunostomy. Resection of the scarred microstomach 
was, probably, an appropriate strategy to prevent possible future complications such as peptic ulcers and malignant transformation.

Early results are promising. We consider resection of a microstomach and fashioning of a Roux-en-Y esophagojejunostomy as components of a safe and good option for the treatment of severe complicated CM.

\section{References}

1 Nagendran S, Johal N, Set P, Brain J, Aslam A, Samuel M. Bilateral communicating intralobar sequestration and microgastria. Ann Thorac Surg 2009; 88:2040.

2 Filippi L, Serafini L, Fiorini P, Agostini E, Giovannucci Uzielli ML. Congenital microgastria and primary ciliary dyskinesia in a newborn with DiGeorge syndrome and 22q11.2 deletion. Eur J Pediatr Surg 2008; 18:195-197.

3 Jones VS, Cohen RC. An eighteen year follow-up after surgery for congenital microgastria-case report and review of literature. J Pediatr Surg 2007; 42:1957-1960.
4 Sharma SC, Menon P. Congenital microgastria with esophageal stenosis and diaphragmatic hernia. Pediatr Surg Int 2005; 21:292-294.

5 Herman TE, Siegel MJ. Imaging casebook. Asplenia syndrome with congenital microgastria and malrotation. J Perinatol 2004; 24:50-52.

6 Menon P, Rao KL, Cutinha HP, Thapa BR, Nagi B. Gastric augmentation in isolated congenital microgastria. J Pediatr Surg 2003; 38:E4-E6.

7 Kroes EJ, Festen C. Congenital microgastria: a case report and review of literature. Pediatr Surg Int 1998; 13:416-418.

8 Langman J. Digestive system. In: Langman J, editor. Medical embryology. Baltimore, MD: Williams \& Wilkins; 1981.pp. 215-216.

9 Lall A, Morabito A, Bianchi A. 'Total Gastric Dissociation (TGD)' in difficult clinical situations. Eur J Pediatr Surg 2006; 16:396-398.

10 Blank E, Chisolm AJ. Congenital microgastria: a case report with a 26-year follow-up. Pediatrics 1973; 51:1037-1041.

11 Kawaguchi AL, Donahoe PK, Ryan DP. Management and long-term follow-up of patients with types III and IV laryngotracheoesophageal clefts. $J$ Pediatr Surg 2005; 40:158-164. [discussion 164-165].

12 Laurie DE, Wakeling EL. Congenital microgastria in association with Pierre-Robin sequence. Clin Dysmorphol 2008; 17:143-144.

13 Waasdorp CE, Rooks V, Sullivan C. Congenital microgastria presenting as stridor. Pediatr Radiol 2003; 33:662-663. 